\title{
Coniferaldehyde prevents articular cartilage destruction in a murine model via $\mathrm{Nrf2/HO-1}$ pathway
}

\author{
DAWEI CAI ${ }^{1 *}$, JIELING WANG ${ }^{2 *}$, SICHUN CHEN $^{1}$, LONGHAI JIANG ${ }^{1}$, \\ JINWEI CHEN $^{1}$, JI WU ${ }^{1}$ and JIAN QIN ${ }^{1}$ \\ ${ }^{1}$ Department of Orthopaedics, Sir Run Run Hospital, Nanjing Medical University, Nanjing, Jiangsu 211100; \\ ${ }^{2}$ Department of Critical Medicine, The Fourth Affiliated Hospital of Anhui Medical University, \\ Hefei, Anhui 230011, P.R. China
}

Received April 28, 2020; Accepted December 17, 2020

DOI: $10.3892 / \mathrm{mmr} .2021 .11863$

\begin{abstract}
Osteoarthritis (OA) is the most prevalent joint disorder characterized by progressive cartilage damage, resulting in gradual disability among the elderly. We previously provided in vivo evidence that nuclear factor erythroid 2-related factor 2 (Nrf2) deficiency is associated with the development of OA. It has been reported that coniferaldehyde (CFA) acts as a potential Nrf2 activator. The aim of the present study was to investigate the protective effects of CFA against osteoarthritis. A murine model of surgical-induced OA was used in the present study and CFA was administered by peritoneal injection every day, and the knee joints were assessed by histological analysis. The results demonstrated that CFA activated the Nrf2 signaling pathway in primary chondrocytes and articular cartilage from the knee joints. Cartilage damage in mice subjected to the destabilization of the medial meniscus was evidently alleviated by CFA treatment. CFA also robustly suppressed apoptosis induced by $\mathrm{H}_{2} \mathrm{O}_{2}$ in murine chondrocytes and reduced the expression of matrix metalloproteinase (MMP)1, MMP3, interleukin (IL)-1 and IL-6 in vivo. On the whole, the findings suggested that CFA exerts a therapeutic effect against OA, and the activation of the Nrf2/heme oxygenase-1 pathway may play a crucial role in CFA-mediated cartilage protection.
\end{abstract}

\section{Introduction}

Osteoarthritis (OA), characterized by progressive cartilage degradation, is the most common joint disorder worldwide (1).

Correspondence to: Professor Jian Qin, Department of Orthopaedics, Sir Run Run Hospital, Nanjing Medical University, 109 Longmian Road, Jiangning, Nanjing, Jiangsu 211100, P.R. China E-mail: qinjian@njmu.edu.cn

${ }^{*}$ Contributed equally

Key words: coniferaldehyde, osteoarthritis, nuclear factor erythroid 2-related factor 2, heme oxygenase-1, cartilage
OA accounts for major pain, and loss of function and disability in adults. As OA is an age-related disease, the rapid increase in the number of older-aged adults in the population has led to increased incidence. Effective disease-modifying drugs are still limited for the treatment of OA (2). The last option for late-stage $\mathrm{OA}$ is the surgical approach of total joint replacement (3). The etiology and underlying mechanisms responsible for the development of OA remain largely unknown. Recent findings have suggested that the overproduction of reactive oxygen species (ROS) and oxidative stress induces chondrocyte senescence/apoptosis, the degradation of the extracellular matrix or synovial inflammation in OA $(4,5)$. Nuclear factor erythroid 2-related factor 2 (Nrf2) has been identified as one of the transcription factors that binds to antioxidant response elements (AREs) to induce the expression of a number of detoxication enzymes, including heme oxygenase-1 (HO-1), which exerts anti-inflammatory effects during OA $(6,7)$. Previously published research from our laboratory demonstrated that deficiency of Nrf2 leads to an increased severity of OA in an inflammatory model and a post-traumatic model of OA (8). Thus, the activation of the Nrf2/ARE signaling pathway offers a novel perspective for the exploration of potential treatment strategies for OA. Coniferaldehyde (CFA), a food flavoring, has been demonstrated to potently activate Nrf2 in RAW264.7 macrophages, exerting cytoprotective effects against lipopolysaccharide-induced cell death (9). The aim of the present study was to investigate whether CFA can exert protective effects against $\mathrm{OA}$ by activating the $\mathrm{Nrf}-2 / \mathrm{HO}-1$ signaling pathway.

\section{Materials and methods}

Chemicals and reagents. CFA (purity, 98\%) was purchased from Sigma-Aldrich (Merck KGaA; cat. no. 382051). Anti-HO-1 (cat. no. BS6626; 1:2,000) and secondary HRP-conjugated goat anti-rabbit (cat. no. BS13278; 1:5,000) were obtained from Bioworld Technology, Inc. Anti-Nrf2 (cat. no. 12721; 1:2,000), anti-lamin B (cat. no. 13435; 1:1,000), Alexa Fluor 488 anti-rabbit IgG (cat. no. 4412; 1:200) and anti- $\beta$-actin (cat. no. 8457; 1:5,000) antibodies were procured from Cell Signaling Technology, Inc. The nuclear protein extraction kit (cat. no. P0028) and Cell Counting Kit-8 (CCK-8; cat. no. C0037) were supplied by Beyotime Institute of 
Biotechnology. The malondialdehyde (MDA; cat. no. A003-4), catalase (CAT; cat. no. A007-2) and superoxidase dismutase (SOD; cat. no. A001-3) assay kits were purchased from Nanjing Jiancheng Bioengineering Institute. The fluorescent probe, 2',7'-dichlorodihydrofluorescein diacetate (H2DCFDA; cat. no. D399), was supplied by Thermo Fisher Scientific, Inc.

Cells and cell culture. Primary mouse chondrocytes were isolated from murine costal cartilage obtained from 29 male neonatal B6 mice (age, 6 days old; weight, 2.92 $\pm 0.46 \mathrm{~g}$ ) purchased from the Comparative Medical Center of Yangzhou University (Yangzhou, China) and were housed under a $12 \mathrm{~h} \mathrm{light/dark}$ cycle at a room temperature of $22 \pm 1^{\circ} \mathrm{C}$ and $50 \%$ relative humidity with food and water available ad libitum, as previously described (10). The animal research was carried out in April 2019 in accordance with Nanjing Medical University Institutional Animal Care and Use Committee guidelines (approval no. IACUC 1901062). Mice were euthanized using $100 \% \mathrm{CO}_{2}$ anesthesia using an air displacement rate of $20 \%$ of the chamber volume $/ \mathrm{min}$. Following digestion with collagenase $\mathrm{D}$ overnight at $37^{\circ} \mathrm{C}$, the collected cells were cultured at a density of $5 \times 10^{5}$ cells/per dish (10 cm in diameter) in Ham's F-12 (DMEM/F12; Gibco; Thermo Fisher Scientific, Inc.) containing $2 \mathrm{mM}$ L-glutamine, 5\% FBS (Gibco; Thermo Fisher Scientific, Inc.), $100 \mathrm{IU} / \mathrm{ml}$ penicillin and $100 \mu \mathrm{g} / \mathrm{ml}$ streptomycin at $37^{\circ} \mathrm{C}$ and $5 \% \mathrm{CO}_{2}$. Morphology observation of chondrocytes with light-contrast microscopes and immunostaining of chondrocytes in cultures with an anti-type II collagen antibody were used to phenotype chondrocytes (data not shown). For immunostaining, the cells was blocked with $3 \%$ goat serum (Invitrogen; Thermo Fisher Scientific, Inc.) for $2 \mathrm{~h}$ at $22^{\circ} \mathrm{C}$, and then incubated with primary antibody against type II collagen (Bioworld Technology, Inc; cat. no. BS1071; $1: 200)$ for $2 \mathrm{~h}$ at room temperature, and were subsequently incubated with fluorescein-conjugated antibody (Cell Signaling Technology, Inc; cat. no. 4412; 1:200) for $1 \mathrm{~h}$ at $22^{\circ} \mathrm{C}$. Primary chondrocytes at $80 \%$ confluency were detached and plated in 6 -well plates $(2.4 \mathrm{~cm}$ in diameter) for use in further assays.

Cell viability assays. The viability of the treated and untreated primary mouse chondrocytes was measured by a CCK-8 assay. Briefly, chondrocytes at passage 1 were incubated in a 12 -well plate at a density of $5 \times 10^{3}$ cells/well. Following $24 \mathrm{~h}$ of incubation with various concentrations of CFA $(0,30$, $60,90$ and $120 \mu \mathrm{M})$ at $37^{\circ} \mathrm{C}, \mathrm{CCK}-8$ working solution was added at $10 \mu \mathrm{l} /$ well followed by further incubation for $1 \mathrm{~h}$ in the dark. The absorbance was monitored at a wavelength of $450 \mathrm{~nm}$ using a microplate reader (Bio-Rad Laboratories, Inc.) according to the manufacturer's protocols.

OA model and histological analysis. A total of 95 B6 male mice (weight, 26.40 $\pm 3.92 \mathrm{~g}$; age, 8-10 weeks old) supplied by the Comparative Medical Center of Yangzhou University (Yangzhou, China) were used in the present study. The mice were anesthetized by administering intraperitoneal ketamine hydrochloride $(120 \mathrm{mg} / \mathrm{kg})$ and xylazine hydrochloride $(5 \mathrm{mg} / \mathrm{kg})$. The levels of anesthesia were considered adequate when the mice stayed still quietly, were unresponsive to external stimuli and had constant heart and respiratory rates. Mice were euthanized using $100 \% \mathrm{CO}_{2}$ anesthesia using an air displacement rate of $20 \%$ of the chamber volume/min. All animal research was executed in accordance with the Nanjing Medical University Institutional Animal Care and Use Committee guidelines. The mice were housed in a normal atmosphere under a 12 -h light-dark cycle at $24^{\circ} \mathrm{C}$ with $50 \%$ humidity, and provided with standard laboratory food (RM3; Special Dietary Systems) and water ad libitum. Mice were divided into four groups [sham surgery + saline, sham surgery + CFA, destabilization of the medial meniscus (DMM) surgery + saline and DMM surgery + CFA). OA was induced by DMM surgery on the right knee joints according to previously reported procedures (11). The delivery of CFA $(0.05 \mathrm{mmol} \mathrm{kg} /$ day) was performed via peritoneal injection at 2 weeks following surgery. The mice were sacrificed at 8 weeks post-OA surgery for histological assessment and WB analysis. Serial sections (thickness, $5 \mu \mathrm{m}$ ) of knee joints were placed in $70 \%$ ethyl alcohol for $15 \mathrm{~min}$. Then, the sections were stained with $0.04 \%$ safranin $\mathrm{O} /$ sodium acetate buffer for $10 \mathrm{~min}$ at room temperature. The severity of cartilage deterioration was evaluated using the 0-6 scoring system recommended by the Osteoarthritis Research Society International (OARSI) with an Olympus BX51 light microscope and photographed by a computer-operated Olympus DP72 digital camera (Olympus Corporation) (12). The observers were blinded to the experimental conditions.

Protein expression analysis via western blotting (WB). The chondrocytes at passage 1 were treated with various concentrations of CFA for $24 \mathrm{~h}$. The collected cells were lysed on ice for $15 \mathrm{~min}$ with pre-cooled RIPA lysis buffer $(50 \mathrm{mM}$ Tris- $\mathrm{HCl}, 150 \mathrm{mM} \mathrm{NaCl}, \mathrm{pH}$ 7.4, 1 mM EDTA, 0.5\% sodium deoxycholate, $1 \%$ Nonidet P40 and $0.1 \%$ SDS). Mice were administered CFA ( $0.05 \mathrm{mmol} \mathrm{kg} /$ day) or saline for 2 weeks or 6 weeks, and the harvested cartilage samples were prepared as previously described (13). Briefly, knee cartilage obtained from mice using a scalpel blade with a surgical microscope was stored in liquid nitrogen. Following pulverization, samples were solubilized at $4^{\circ} \mathrm{C}$ with lysis buffer for $30 \mathrm{~min}$. For WB analysis, both cell and tissue samples were centrifuged at $12,000 \mathrm{x} \mathrm{g}$ for $20 \mathrm{~min}$ at $4^{\circ} \mathrm{C}$, and the supernatant was collected to determine the protein content with a BCA protein quantitation kit (Pierce; Thermo Fisher Scientific, Inc.). Proteins of the same quality were loaded on $10 \%$ gels and separated via SDS-PAGE, and subsequently separated proteins were transferred onto PVDF membranes. The membranes were blocked with $5 \%$ milk at room temperature for $1 \mathrm{~h}$, and then probed with the desired primary antibodies (anti-Nrf2, anti-HO-1, anti-lamin B and anti- $\beta$-actin) at $4^{\circ} \mathrm{C}$ overnight. Following incubation with primary antibodies, the membranes were washed three times in Tris-buffered saline with $0.1 \%$ Tween-20 (TBST), then detected with secondary HRP-conjugated goat anti-rabbit antibodies at room temperature for $1 \mathrm{~h}$. The membranes were finally washed three times in TTBS, and were then visualized by ECL High-Signal reagent (Thermo Fisher Scientific, Inc.) and analyzed with ImageJ software (version 1.43, National Institutes of Health) for semiquantification. To obtain a suitable amount of protein, the pooling of the samples was performed; each experimental unit for mice comprised a pool of two to three compartments; 
Table I. Gene specific primer sequences for reverse transcription-quantitative PCR.

\begin{tabular}{ll}
\hline Genes & \multicolumn{1}{c}{ Primer sequences $\left(5^{\prime} \rightarrow 3^{\prime}\right)$} \\
\hline IL-1 & F: ATGGCAGAAGTACCTAAGCTCGC \\
IL-6 & R: ACACAAATTGCATGGTGAAGTCAGTT \\
& F: ACACACTGGTTCTGAGGGAC \\
TNF- $\alpha$ & R: TACCACAAGGTTGGCAGGTG \\
& F: ATGAGCACAGAAAGCATGATCCGC \\
MMP1 & R: CCAAAGTAGACCTGCCCGGACTC \\
MMP3 & F: GCCACAAAGTTGATGCAGTT \\
& R: GCAGTTGAACCAGCTATTAG \\
MMP13 & F: ATGAAAATGAAGGGTCTTCCGG \\
& R: GCAGAAGCTCCATACCAGCA \\
ACTB & F: ATGCATTCAGCTATCCTGGCCA \\
& R: AAGATTGCATTTCTCGGAGCCTG \\
& F: TGACGGGGTCACCCACACTGTGCCCATCTA \\
& R: CTAGAAGCATTTGCGGTGGACGATGGAGGG
\end{tabular}

F, forward; R, reverse; MMP, matrix metalloproteinase; IL-, interleukin; ACTB, actin.

when pooling was performed, the experimental unit was regarded as one.

Gene transcript analysis via reverse transcription-quantitative PCR (RT-qPCR). Cartilage was procured and snap-frozen in liquid nitrogen prior to RNA extraction. Pooling was also performed to obtain a suitable amount of cartilage and each experimental unit comprised a pool of two compartments. Total RNA extracted from the cartilage in the knee joints from the mice was isolated using TRIzol ${ }^{\circledR}$ reagent (Invitrogen; Thermo Fisher Scientific, Inc.). cDNA was synthesized from total RNA using the PrimeScript RT Reagent kit (Takara Bio, Inc.), according to the manufacturer's protocol. PCR thermocycling conditions were as follows: Initial denaturation at $96^{\circ} \mathrm{C}$ for $10 \mathrm{~min}$, followed by 30 cycles of denaturation at $95^{\circ} \mathrm{C}$ for $15 \mathrm{sec}$, annealing and elongation at $60^{\circ} \mathrm{C}$ for $30 \mathrm{sec}$; and final extension at $72^{\circ} \mathrm{C}$ for $10 \mathrm{~min}$. qPCR was performed on a 7500 real-time PCR system using SYBR-Green PCR Master Mix (Thermo Fisher Scientific, Inc.) and repeated in triplicate for each sample. Primer pairs used in the present study are listed in Table I. Relative quantification was performed using the $2^{-\Delta \Delta \mathrm{Cq}}$ method (14).

Luciferase assays. The HO-1 promoter was amplified by general PCR and the product was subsequently inserted into the pGL3 vector (Promega Corporation) at the HindIII and BglII sites. A non-specific oligonucleotide was used to construct a control plasmid. The DNA was extracted from RAW cells using the TIANamp Genomic DNA kit (cat. no. DP304; Tiangen Biotech Co., Ltd.) and amplified with AmpliTaq Gold ${ }^{\mathrm{TM}}$ DNA Polymerase (Thermo Fisher Scientific, Inc.) following the manufacturer's instructions. The primer sequences were as follows: HO-1 forward, 5'-GGAAGATCTCTGCAGAGCCCC ACTGGAG-3' and reverse, 5'-CCCAAGCTTGGAACAGCA ACGCTGT-3'. The thermocycling conditions were as follows:
$2 \mathrm{~min}$ at $94^{\circ} \mathrm{C} ; 30$ cycles of $30 \mathrm{sec}$ at $94^{\circ} \mathrm{C}, 30 \mathrm{sec}$ at $56^{\circ} \mathrm{C}$ and $30 \mathrm{sec}$ at $72^{\circ} \mathrm{C}$, and a final $5 \mathrm{~min}$ at $72^{\circ} \mathrm{C}$. The 293 cells $\left(5 \times 10^{3}\right.$ cells/well) obtained from the Cell Bank of the Type Culture Collection of the Chinese Academy of Science (China) were transfected with the plasmid (plasmid DNA $1 \mu \mathrm{g}$ ) using Lipofectamine ${ }^{\circledR} 2000$ reagent (Invitrogen; Thermo Fisher Scientific, Inc.) according to the manufacturer's instructions. Following $24 \mathrm{~h}$ of transfection, the cells were treated with 0 , 50 and $100 \mu \mathrm{M} \mathrm{CFA}$ for $24 \mathrm{~h}$ at $37^{\circ} \mathrm{C}$, and a luciferase assay system (Promega Corporation) was used to measure the luciferase activity by normalizing the Firefly luciferase activity to Renilla luciferase activity.

Immunofluorescence staining. Primary chondrocytes were seeded on glass coverslips in a 6-well plate $(2.4 \mathrm{~cm}$ in diameter; $1 \times 10^{5}$ cells/well) and treated with CFA $(100 \mu \mathrm{M})$ for $24 \mathrm{~h}$. Following the rinsing of the glass coverslips with ice-cold PBS three times, the cells were fixed in $4 \%$ paraformaldehyde for $15 \mathrm{~min}$ at $22^{\circ} \mathrm{C}$ and subsequently permeabilized in PBS containing 2\% Triton X-100 for $15 \mathrm{~min}$ at room temperature. After the cells were blocked with $3 \%$ goat serum (Invitrogen; Thermo Fisher Scientific, Inc.) for $90 \mathrm{~min}$ at $22^{\circ} \mathrm{C}$, the cells were incubated with primary antibody against Nrf2 in blocking medium for $2 \mathrm{~h}$ at room temperature, and were subsequently incubated with fluorescein-conjugated antibody (Cell Signaling Technology, Inc; cat. no. 4412; 1:200) for $1 \mathrm{~h}$ at $22^{\circ} \mathrm{C}$. After washing with PBS, the cells were labeled with DAPI (Invitrogen; Thermo Fisher Scientific, Inc.) for $15 \mathrm{~min}$ at room temperature. The stained cells were observed under a confocal microscope (Zeiss LSM 710 META; Carl Zeiss AG).

Apoptosis detection by flow cytometry. The apoptosis of murine primary chondrocytes was measured by Annexin-V-FITC/PI staining assay (Thermo Fisher Scientific, Inc.). Primary chondrocytes cultured in 12-well 
plates $\left(5 \times 10^{3}\right.$ cells/well) subjected to different treatments were stained with $5 \mu \mathrm{l}$ Annexin V-FITC and $5 \mu \mathrm{l}$ PI. The mixture was incubated for $15 \mathrm{~min}$ at room temperature in the dark. The percentage of apoptotic cells (Annexin $\mathrm{V}-\mathrm{FITC}^{+} / \mathrm{PI}$ ) was calculated using a flow cytometer (BD Biosciences) according to the manufacturer's instructions. Data were acquired via flow cytometry (Beckman Coulter, Inc.). Fluorescence analyses were performed on COULTER EPICS XL Flow Cytometer (Beckman Coulter, Inc.) using Expo32-ADC software (version 1.2B; Beckman Coulter, Inc.).

Detection of intracellular ROS and measurement of antioxidant enzyme activities. The intracellular ROS levels were determined with the use of the fluorescent probe, $\mathrm{H}_{2}$ DCFDA. The cells were treated or not with CFA $(100 \mu \mathrm{M})$ for $24 \mathrm{~h}$, followed by incubation with or without $\mathrm{H}_{2} \mathrm{O}_{2}(40 \mathrm{ng} / \mathrm{ml})$ for a further $24 \mathrm{~h}$ at room temperature. After discarding the medium, the chondrocytes were incubated for $25 \mathrm{~min}$ with $\mathrm{H}_{2}$ DCFDA $(10 \mu \mathrm{M})$ in the dark at room temperature. A flow cytometer (BD Biosciences) was used to monitor the intracellular accumulation of ROS. Data were acquired by flow cytometry (Beckman Coulter, Inc.). Fluorescence analyses were performed on Coulter Epics XL Flow Cytometer (Beckman Coulter, Inc.) using Expo32-ADC software. In order to monitor the activities of antioxidant enzymes, the treated cells were homogenized and centrifuged at $13,000 \mathrm{x} \mathrm{g}$ for $5 \mathrm{~min}$ at $4^{\circ} \mathrm{C}$ for supernatants. The levels of SOD, MDA and CAT in the supernatants were measured according to the instructions provided with the respective kits (Nanjing Jiancheng Bioengineering Institute). All the analyses were performed in six samples, each of them in triplicate.

Statistical analysis. All data are expressed as the mean \pm SD. All assays were repeated at least three times independently. The Mann-Whitney U test was used to assess independent pairs, and one-way analysis of variance followed by Tukey's post hoc test were used to assess multiple samples. Statistical analyses were conducted using SPSS software version 11.5 (SPSS, Inc.). $\mathrm{P}<0.05$ was considered to indicate a statistically significant difference.

\section{Results}

CFA activates Nrf2 in primary chondrocytes and knee cartilage. The cytotoxicity of CFA was analyzed in primary murine chondrocytes and concentrations $<120 \mu \mathrm{M}$ were used in subsequent assays (Fig. 1A). CFA treatment led to a significant increase in luciferase activity in a dose-dependent manner (Fig. 1B). The results of the immunofluorescence assays revealed that CFA treatment promoted the nuclear translocation of Nrf2 (Fig. 1C). Consistently, nuclear protein was extracted and assayed for Nrf2 by WB analysis. CFA significantly upregulated the expression of nuclear Nrf2 (Fig. 1D and H). The protein expression of Nrf2 and its downstream protein, HO-1, in primary mouse chondrocytes steadily increased following CFA treatment in a dose- and time-dependent manner (Fig. 1E, F and I-L). To investigate the effects of CFA on Nrf2 and HO-1 expression in the cartilage of knee joints, mice were treated with CFA $(0.2 \mathrm{mmol} \mathrm{kg} / \mathrm{day})$ via gastric gavage for 2 weeks and cartilage samples were harvested for use in WB analysis. The results revealed that expression of Nrf2 and HO-1 in cartilage were significantly higher in the CFA-treated group (Fig. 1G, M and N).

CFA regulates the activities of antioxidant markers and apoptosis in chondrocytes. In order to investigate the antioxidative effects of CFA in primary chondrocytes, oxidative stress was determined by measuring the activities of CAT, MDA and SOD, and the level of intracellular ROS. The $\mathrm{H}_{2} \mathrm{O}_{2}$-induced increase in the MDA and ROS levels was significantly inhibited following $24 \mathrm{~h}$ of CFA treatment (Fig. 2A and B). In addition, the suppressed activity of SOD and CAT was evidently restored by treatment with CFA (Fig. 2C and D). These results suggested that CFA exerted a potent ROS-eliminating effect, protecting primary chondrocytes against oxidative stress-induced damage. It was also found that CFA reversed the decrease in cell viability and the increase in the apoptotic rate, which were induced by exposure of the cells to $\mathrm{H}_{2} \mathrm{O}_{2}$ (Fig. 2E and F). This suggested that CFA exerted protective effects against cartilage damage by scavenging the overproduction of ROS, which causes the apoptosis of chondrocytes.

CFA alleviates cartilage destruction in experimental OA. To determine the protective effects of CFA on OA, a murine model of OA was established by transecting the medial meniscotibial ligament of mice, followed by treatment with either CFA $(0.05 \mathrm{mmol} / \mathrm{kg} / \mathrm{day})$ or the same amount of the vehicle for 6 weeks (Fig. 3C). Histological sections stained with Safranin $\mathrm{O} /$ Fast Green were assessed using OARSI scores in a blinded manner. The vehicle-treated (OA + saline) group displayed notable proteoglycan loss, and cartilage erosion or cartilage damage at 8 weeks post-DMM surgery (Fig. 3A). The OARSI scores of the vehicle-treated group reached as high as $2.63 \pm 0.10$ for the femur and $4.40 \pm 1.33$ for the tibia, while the CFA-treated group exhibited significantly improved scores, with $1.20 \pm 0.85$ for the femur and $3.03 \pm 1.45$ for the tibia (Fig. 3B). These data demonstrated that CFA suppressed the observed degradation of cartilage, thus attenuating the progression of OA.

CFA promotes the expression of $\mathrm{Nrf} 2 / \mathrm{HO}-1$ in experimental $O A$. The levels of Nrf2 and HO-1 in the cartilage from knee joints were measured by WB analysis to ascertain whether the regulation of Nrf2/HO-1 in response to CFA was the same as that observed in vitro. To obtain sufficient proteins for WB analysis, pooling from 32 joints was performed. The results revealed that the expression levels of Nrf2/HO-1 were upregulated in the cartilage from the knee joints of the murine model of OA induced by surgery following the systematic oral administration of CFA (Fig. 4A-C), suggesting that CFA may confer protection against $\mathrm{OA}$ in mice via the activation of the Nrf2/HO-1 pathway.

CFA regulates $O A$-associated $m R N A$ expression. To further elucidate the mechanisms underlying the chondroprotective effects of CFA in vivo, the effects of CFA on the expression of inflammatory and catabolic factors in articular cartilage were further explored. The results revealed that the protein expression levels of matrix metalloproteinase (MMP)1, MMP13, TNF- $\alpha$ and interleukin (IL)-1 were significantly increased in the OA group compared with the control group, and the 

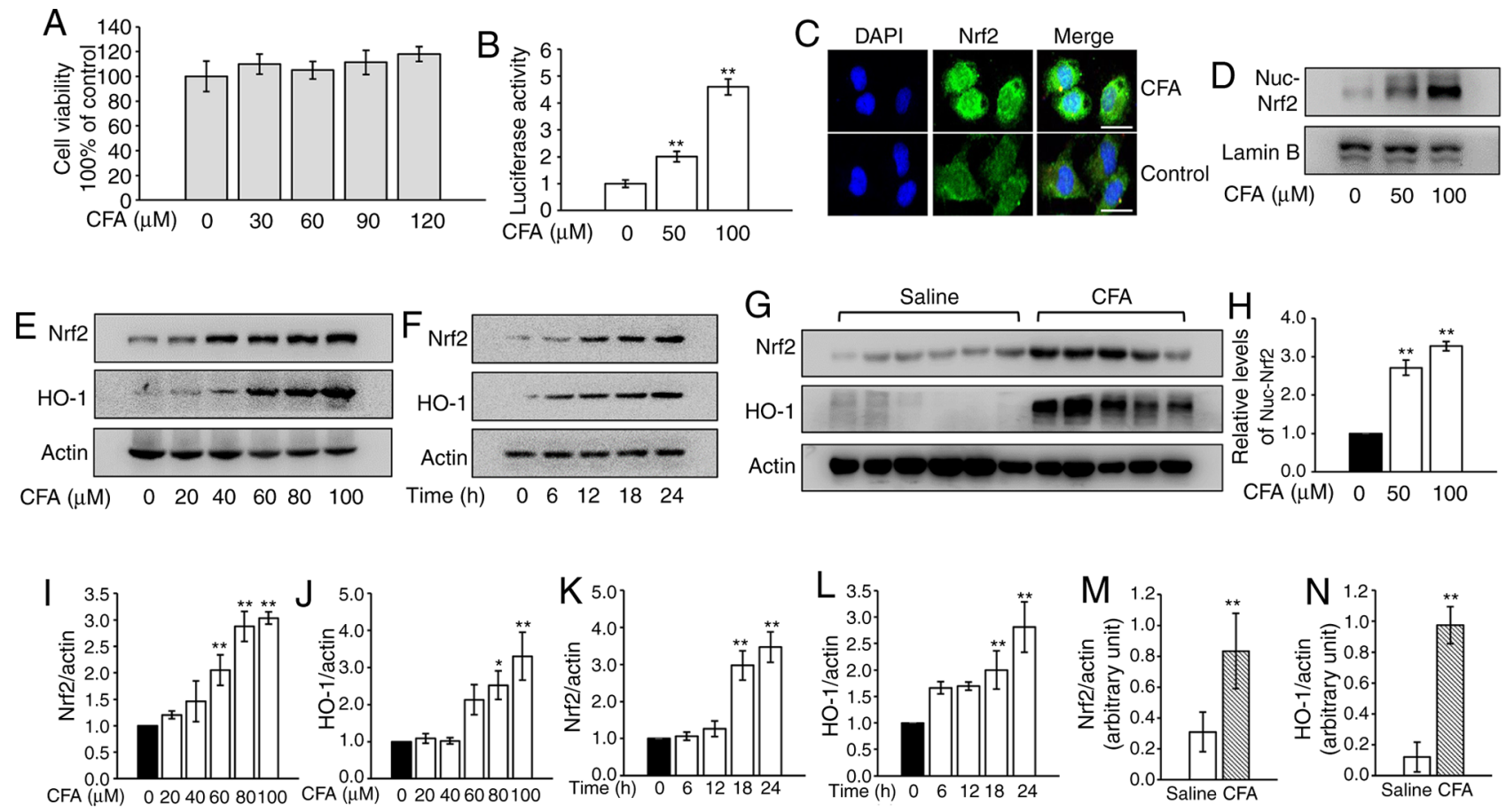

Figure 1. CFA activates the Nrf2 signaling pathway in vitro and in vivo. (A) Cell viability assay. Primary chondrocytes were treated with various concentrations of CFA for $24 \mathrm{~h}$ and analyzed using a Cell Counting Kit-8 assay in triplicate. (B) Luciferase assay. 293 cells were transfected with the HO-1 promoter reporter plasmid and a Renilla luciferase plasmid, followed by treatment with increasing concentrations of CFA for $24 \mathrm{~h}$. The luciferase activity was measured and normalized to Renilla luciferase activity. ${ }^{* *} \mathrm{P}<0.01$ vs. untreated control. (C) The nuclear translocation of Nrf2 was detected by immunofluorescence combined with DAPI staining for nuclei. Scale bar, $100 \mu \mathrm{m}$. (D) Primary murine chondrocytes were treated with CFA for $24 \mathrm{~h}$. The cell nuclear fractions were isolated and assayed for Nrf2 by WB analysis. Lamin B served as a control. (E) Primary murine chondrocytes were treated with various concentrations of CFA for $24 \mathrm{~h}$, and Nrf2 and HO-1 were assayed by WB analysis. (F) Primary murine chondrocytes were treated with CFA (100 $\mu$ M) for various periods of time. Nrf2 and HO-1 were assayed by WB analysis. (G) A total of 22 knees harvested from 11 mice were used for WB analysis of Nrf2 and HO-1 in the cartilage of knee joints from the saline-treated and CFA-treated groups. (H) Semiquantitative analysis of nuclear Nrf2. ${ }^{* *} \mathrm{P}<0.01$ vs. untreated control. Semiquantitative analysis of (I) Nrf2 and (J) HO-1 protein levels. . $\mathrm{P}<0.05$ and ${ }^{* * *} \mathrm{P}<0.01$ vs. untreated control. Semiquantitative analysis of (K) Nrf2 and (L) HO-1 protein levels. ${ }^{* *} \mathrm{P}<0.01$ vs. untreated control. Semiquantitative analysis of (M) Nrf2 and (N) HO-1 protein levels. ${ }^{* *} \mathrm{P}<0.01$ vs. saline-treated group. CFA, coniferaldehyde; Nrf2, nuclear factor erythroid 2-related factor 2; HO-1, heme oxygenase 1; WB, western blotting.

A
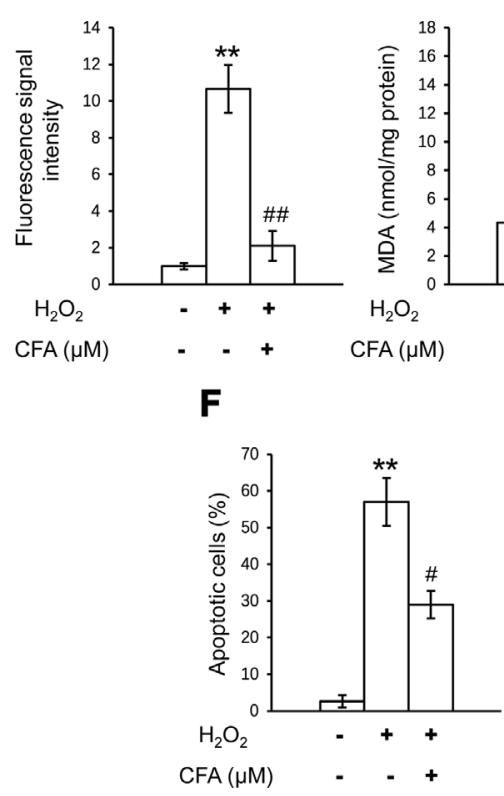

C

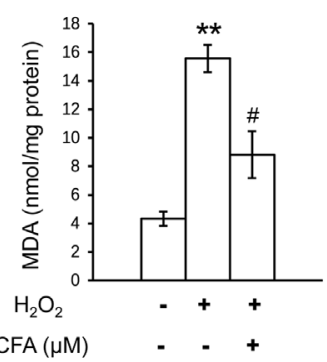

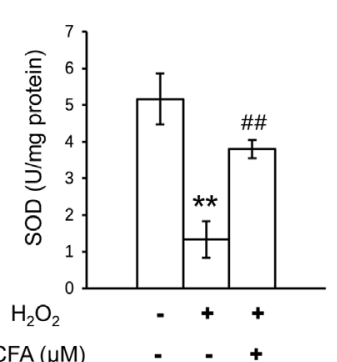

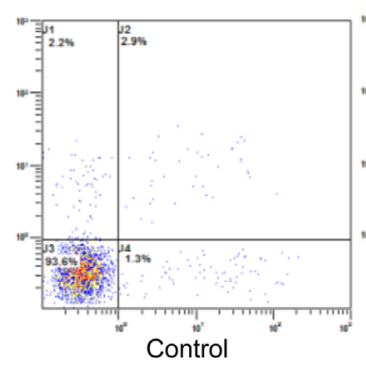

D

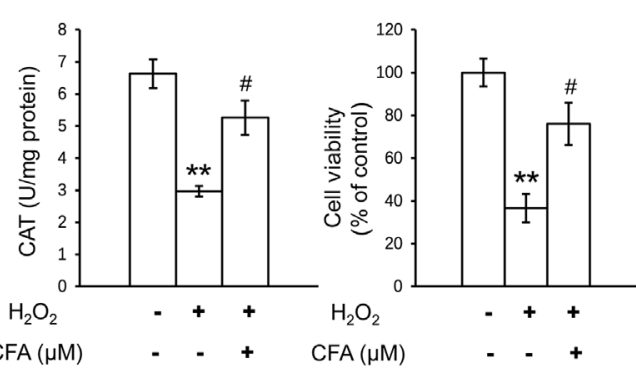

Figure 2. CFA attenuates oxidative stress in primary chondrocytes. Primary murine chondrocytes were pre-treated with $\mathrm{CFA}(100 \mu \mathrm{M})$ for $24 \mathrm{~h}$ and incubated with $40 \mathrm{ng} / \mathrm{ml} \mathrm{H}_{2} \mathrm{O}_{2}$ for $24 \mathrm{~h}$. (A) Effect of CFA treatment on the level of intracellular ROS. (B) Effects of CFA on MDA content. (C) Effects of CFA on SOD activity. (D) Effects of CFA on CAT activity. (E) Cell viability assay. Treated primary chondrocytes were analyzed using a Cell Counting Kit-8 assay in triplicate. (F) Apoptosis of chondrocytes was assessed using a flow cytometer in triplicate. ${ }^{* *} \mathrm{P}<0.01$ vs. untreated control; ${ }^{\#} \mathrm{P}<0.05,{ }^{\# \#} \mathrm{P}<0.01$ vs. $\mathrm{H}_{2} \mathrm{O}_{2}$-exposed group. CFA, coniferaldehyde; ROS, reactive oxygen species; MDA, malondialdehyde; SOD, superoxidase dismutase; CAT, catalase. 


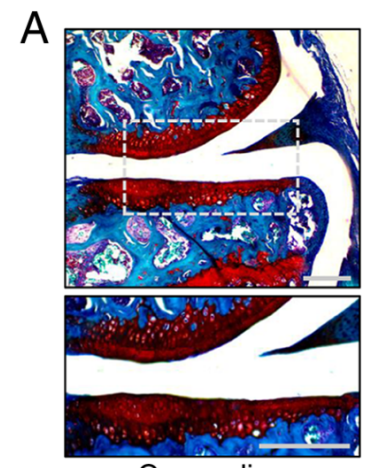

Con+saline

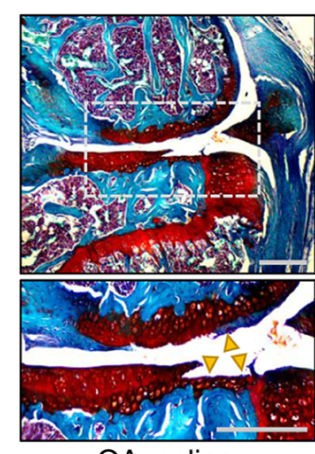

OA+saline
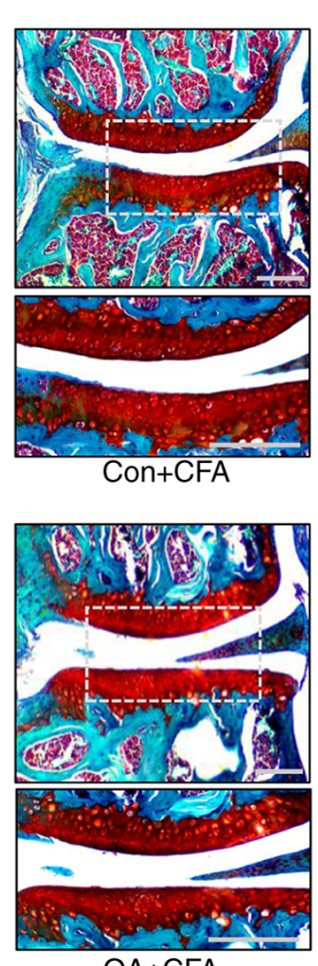
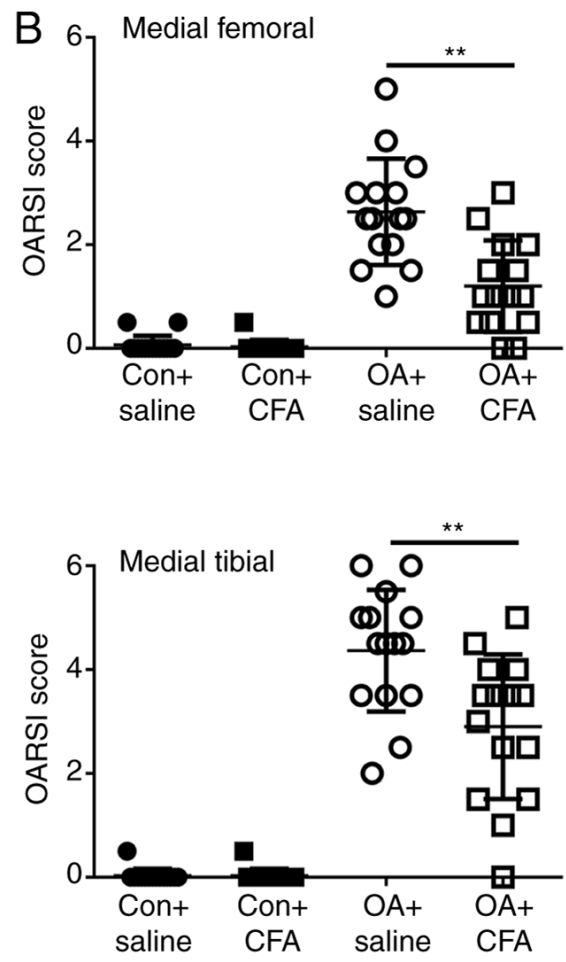

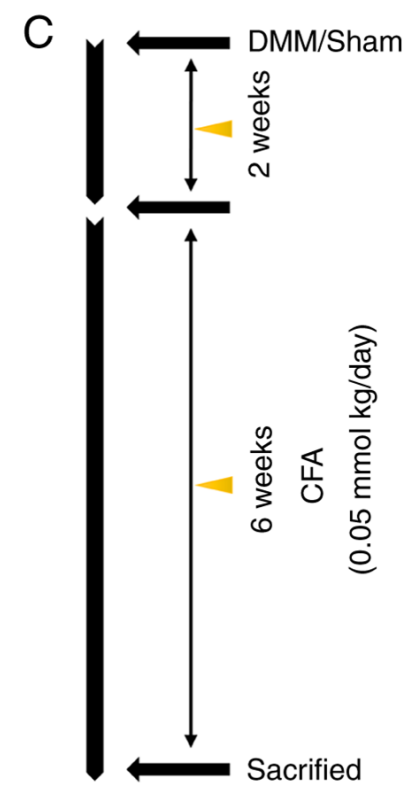

Sacrified

Figure 3. CFA protects against OA. A total of 60 mice subjected to surgery (the right knees were subjected to DMM surgery, the left knees were subjected to sham surgery) were administered CFA or vehicle (saline) at 2 weeks after surgery ( $\mathrm{n}=15 /$ group). (A) Representative sections of knee joints from experimental mice were stained with Safranin O/Fast Green. Yellow triangles indicate the destruction-affected cartilage areas. Scale bar, $100 \mu \mathrm{m}$. (B) Histological scoring of OA from (A). (C) Schematic diagram depicting the time of treatment and the follow-up period of the animals. Mice were administered with CFA or saline.

${ }^{* *} \mathrm{P}<0.01$. CFA, coniferaldehyde; OA, osteoarthritis; DMM, destabilization of the medial meniscus; OARSI, Osteoarthritis Research Society International.
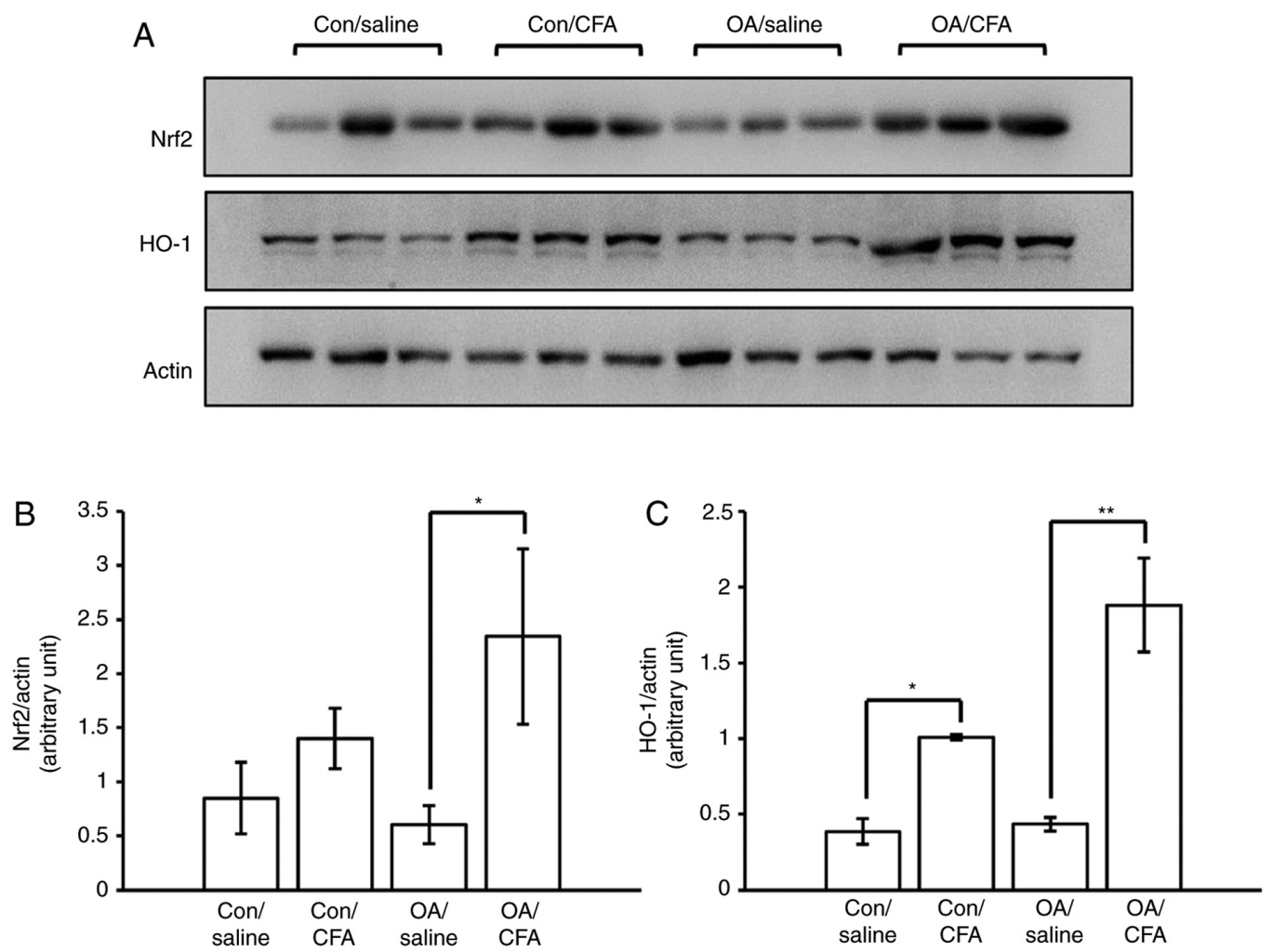

Figure 4. Effects of CFA on the expression of Nrf2/HO-1 in mice with experimental OA. A total of 24 knees harvested from 12 mice were used for western blot analysis. There were a total of four groups: Control knees from saline-treated mice, control knees from CFA-treated mice, OA knees from saline-treated mice, OA knees from CFA-treated mice. (A) Western blot analysis of Nrf2 and HO-1 in the cartilage of murine knee joints. Semiquantitative analysis of (B) Nrf2 and (C) HO-1 protein expression. ${ }^{*} \mathrm{P}<0.05,{ }^{* *} \mathrm{P}<0.01$. CFA, coniferaldehyde; Nrf2, nuclear factor erythroid 2-related factor 2; HO-1, heme oxygenase 1; OA, osteoarthritis. 
A

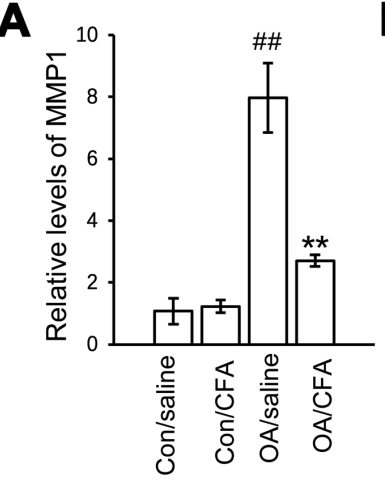

D

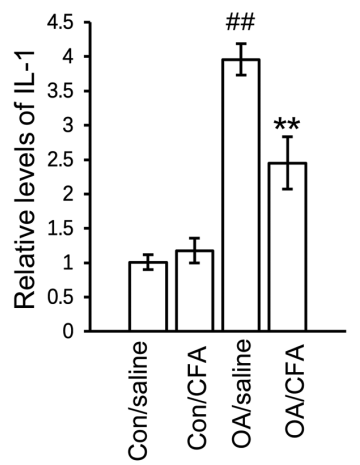

B

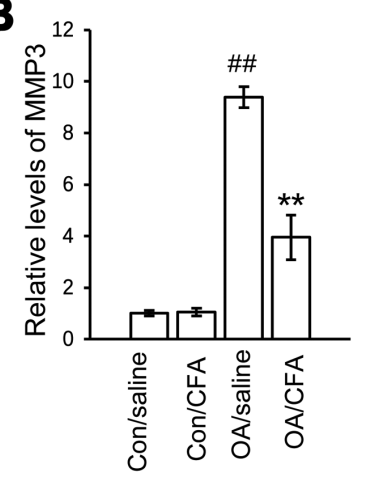

E

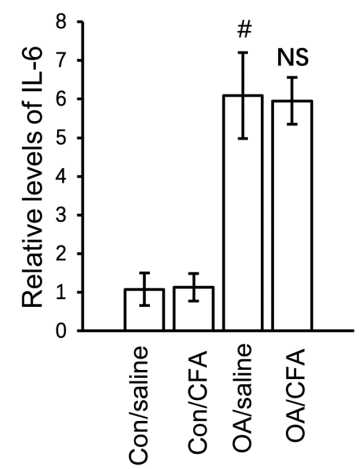

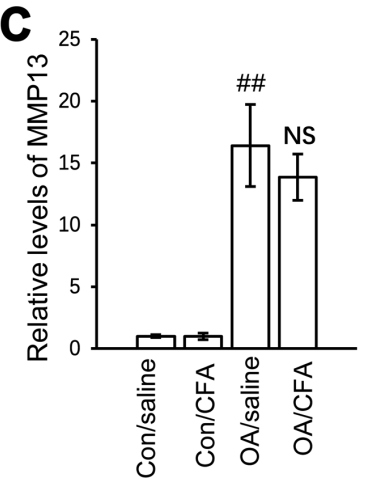

F

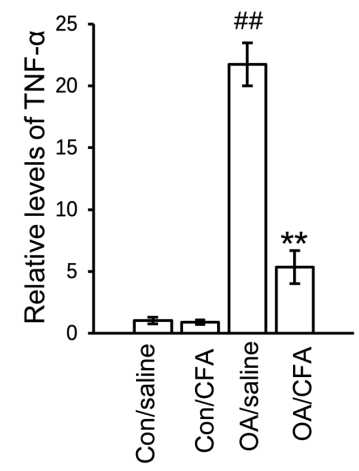

Figure 5. Effects of CFA on the expression of OA-related genes in experimental osteoarthritis. A total of $24 \mathrm{knees} \mathrm{harvested} \mathrm{from} 12$ mice were used for gene transcript analysis. There were a total of four groups: Control knees from saline-treated mice, control knees from CFA-treated mice, OA knees from saline-treated mice, OA knees from CFA-treated mice. mRNA expression of (A) MMP1, (B) MMP3, (C) MMP13, (D) IL-1, (E) IL-6 and (F) TNF- $\alpha$ in the cartilage from the four groups of knee joints quantified by reverse transcription-quantitative $\mathrm{PCR} .{ }^{\#} \mathrm{P}<0.05,{ }^{\# \#} \mathrm{P}<0.01 \mathrm{vs}$. con/saline-treated mice; ${ }^{* *} \mathrm{P}<0.01 \mathrm{vs}$. OA/saline-treated mice. CFA, coniferaldehyde; OA, osteoarthritis; MMP, matrix metalloproteinase; IL-, interleukin.

upregulated gene expression was suppressed, while the expression levels of both MMP3 and IL-6 were not regulated by CFA (Fig. 5A-F).

\section{Discussion}

Nrf2 is a crucial transcriptional regulator mediating protection against oxidative stress. In the resting state, Nrf2 is tethered to its inhibitor in the cytoplasm, Kelch-like ECH-associated protein 1 (Keap1), which regulates its proteasomal degradation (15). When exposed to oxidative stress, Nrf2 is released from Keap1 and translocates to the nucleus, binding to the antioxidant response elements (AREs) located in the promoter regions to regulate the expression of a variety of antioxidant enzymes, thereby providing inducible antioxidant defense. The CFA-induced nuclear translocation of Nrf2, the activation of the HO-1 promoter transactivation activity and the upregulation of its downstream gene expression were all observed in the present study, which indicated that CFA activated the Nrf2 signaling pathway in vivo and in vitro.

Trichostatin A (a histone deacetylase inhibitor) was previously used to induce Nrf2 activation and HO-1 expression, subsequently resulting in the decreased severity of cartilage destruction in mice with OA (8). However, considering the side-effects caused by trichostatin A, potential drugs targeting Nrf2 activation are required. Dong et al (16) treated mice with CFA $(0.2 \mathrm{mmol} / \mathrm{kg} /$ day $)$ orally for $>6$ months and the results of immunohistochemistry indicated that CFA upregulated Nrf2 expression in brain tissues. Since drugs given by oral route usually have low bioavailability due to first pass metabolism, the dose of CFA was adjusted for peritoneal route of administration in the present study. The activation of Nrf2 was observed by WB analysis in the cartilage from the knee joints of mice that were administered CFA $(0.05 \mathrm{mmol} / \mathrm{kg} /$ day $)$ by peritoneal injection for 2 weeks. These results demonstrated that Nrf2 activation serves as an important mechanism for the chondroprotective effects of CFA.

Notably, CFA has been reported to induce the growth of SH-SY5Y cells within the range of $30-160 \mu \mathrm{M}$ (16). The present study evaluated cell viability using a CCK-8 assay, and no increase in cell viability was observed with concentrations between 0 and $120 \mu \mathrm{M}$ CFA (Fig. 1A). These differing results may depend on the cell type used. ROS are usually considered to be a toxic by-product of cell metabolism. A related study found that increased mitochondrial ROS production can trigger chondrocyte death and promote the expression of pro-inflammatory cytokines, leading to the onset and progression of OA (17). On the other hand, the inhibition of ROS production can attenuate the degeneration of cartilage in OA $(18,19) . \mathrm{H}_{2} \mathrm{O}_{2}$ is one of the main types of ROS, which are spontaneously converted to the highly reactive hydroxyl radicals, inducing DNA damage and cell death. In the present study, chondrocytes exposed to $\mathrm{H}_{2} \mathrm{O}_{2}$ exhibited a significant increase in apoptosis with a decreased cell viability. Treatment with CFA alleviated $\mathrm{H}_{2} \mathrm{O}_{2}$-induced apoptosis and increased the viability of primary chondrocytes in the present study, improving mitochondrial function by eliminating the overproduction of ROS induced by $\mathrm{H}_{2} \mathrm{O}_{2}$, and thereby conferring protection against oxidative stress during $\mathrm{OA}$. 
Although there was no significant statistical difference in the expression of both Nrf2 and HO-1 between the OA/CFA group and the Control/CFA group (may be due to the limited number of samples), it was speculated that the level of HO-1, a member of heat shock protein (HSP) family, which is produced by cells in response to exposure to stressful conditions, may be much higher in the OA/CFA group compared with the Control/CFA group. In our previous study, it was found that the level of HO-1 in knee cartilage significantly increased in both patients with OA and DMM-induced OA mice relative to controls, which may be regarded as a beneficial adaptive response to oxidative stress in chondrocytes during the progression of OA (20). These findings suggested that the development of OA also contributed to the increased expression of HO-1 in the OA/CFA group, and the very obvious upregulation of HO-1 induced by CFA may play a key role in delaying the progression of $\mathrm{OA}$.

$\mathrm{HO}$ degrades heme to free iron, biliverdin and carbon monoxide (21). The authors previously reported that HO-1 expression is increased in cartilage from patients diagnosed with OA, which is regarded as an adaptive response against stress during cartilage damage (20). The induction of HO-1 suppresses the IL-1-induced expression of MMPs and inflammatory factors $(22,23)$, which are abnormally enhanced in OA-affected cartilage. In agreement with these studies, the present study found that the expression of several matrix-degrading enzyme genes, including MMP1, MMP3, IL-1 and TNF- $\alpha$, were significantly inhibited in cartilage from the mice subjected to DMM surgery at 8 weeks post-CFA treatment. Although a previous study stated that the upregulation of HO-1 induced by cobalt protoporphyrin IX suppressed the expression of IL-6 in human tracheal smooth muscle cells (24), the present study did not observe a significant effect of CFA induced-HO-1 on the regulation of IL- 6 in cartilage. On the whole, this evidence suggested that CFA also plays an important regulatory role in anti-inflammation and anti-catabolism during OA.

To the best of our knowledge, the present study is the first to demonstrate the cartilage-protective role of CFA in an animal model of OA. CFA activated the Nrf2/HO-1 signaling pathway to exert potent anti-apoptotic and anti-inflammatory effects on cartilage. The present study suggested that the use of CFA may represent a promising treatment strategy for OA.

\section{Acknowledgements}

Not applicable.

\section{Funding}

The present study was subsidized by a grant from the Natural Science Foundation of the Jiangsu Higher Education Institutions of China (grant no. 18KJB320009) for financial support.

\section{Availability of data and materials}

The datasets used and/or analyzed during the current study are available from the corresponding author on reasonable request.

\section{Authors' contributions}

DC, JW and JQ conceived and designed the study. DC, JW, SC, LJ, JC and JW performed the experiments. DC and JW confirm the authenticity of all the raw data. DC and JW wrote the manuscript. JQ reviewed and edited the manuscript. All authors read and approved the manuscript.

\section{Ethics approval and consent to participate}

All experiments related to the use of animals were approved (approval no. IACUC 1901062) by the Institutional Animal Care and Use Committee of The Sir Run Run Hospital of Nanjing Medical University (Nanjing, China).

\section{Patient consent for publication}

Not applicable.

\section{Competing interests}

The authors declare that they have no competing interests.

\section{References}

1. Bihlet AR, Byrjalsen I, Bay-Jensen AC, Andersen JR, Christiansen C, Riis BJ and Karsdal MA: Associations between biomarkers of bone and cartilage turnover, gender, pain categories and radiographic severity in knee osteoarthritis. Arthritis Res Ther 21: 203, 2019.

2. Wang Y, Hussain SM, Wluka AE, Lim YZ, Abram F, Pelletier JP, Martel-Pelletier J and Cicuttini FM: Association between metformin use and disease progression in obese people with knee osteoarthritis: Data from the osteoarthritis Initiative-a prospective cohort study. Arthritis Res Ther 21: 127, 2019.

3. Xu H, Zhao G, Xia F, Liu X, Gong L and Wen X: The diagnosis and treatment of knee osteoarthritis: A literature review. Int J Clin Exp Med 12: 4589-4599, 2019.

4. Lepetsos P and Papavassiliou AG: ROS/oxidative stress signaling in osteoarthritis. Biochim Biophys Acta 1862: 576-591, 2016.

5. Khan NM, Haseeb A, Ansari MY, Devarapalli P, Haynie S and Haqqi TM: Wogonin, a plant derived small molecule, exerts potent anti-inflammatory and chondroprotective effects through the activation of ROS/ERK/Nrf2 signaling pathways in human osteoarthritis chondrocytes. Free Radic Biol Med 106: 288-301, 2017.

6. Loboda A, Damulewicz M, Pyza E, Jozkowicz A and Dulak J: Role of Nrf2/HO-1 system in development, oxidative stress response and diseases: An evolutionarily conserved mechanism. Cell Mol Life Sci 73: 3221-3247, 2016.

7. Zhao K, Li Y, Wang Z, Han N and Wang Y: Carnosine protects mouse podocytes from high glucose induced apoptosis through PI3K/AKT and Nrf2 pathways. Biomed Res Int 2019: 4348973, 2019.

8. Cai D, Yin S, Yang J, Jiang Q and Cao W: Histone deacetylase inhibition activates Nrf2 and protects against osteoarthritis. Arthritis Res Ther 17: 269, 2015.

9. Kim KM, Heo DR, Kim YA, Lee J, Kim NS and Bang OS: Coniferaldehyde inhibits LPS-induced apoptosis through the PKC $\alpha / \beta$ II/Nrf-2/HO-1 dependent pathway in RAW264.7 macrophage cells. Environ Toxicol Pharmacol 48: 85-93, 2016.

10. Gosset M, Berenbaum F, Thirion S and Jacques C: Primary culture and phenotyping of murine chondrocytes. Nat Protoc 3: 1253-1260, 2008.

11. Glasson SS, Blanchet TJ and Morris EA: The surgical destabilization of the medial meniscus (DMM) model of osteoarthritis in the 129/SvEv mouse. Osteoarthritis Cartilage 15: 1061-1069, 2007.

12. Glasson SS, Chambers MG, Van Den Berg WB and Little CB: The OARSI histopathology initiative-recommendations for histological assessments of osteoarthritis in the mouse. Osteoarthritis Cartilage 18 (Suppl 3): S17-S23, 2010. 
13. Cai D, Feng W, Liu J, Jiang L, Chen S, Yuan T, Yu C, Xie H, Geng D and Qin J: 7,8-Dihydroxyflavone activates Nrf2/HO-1 signaling pathways and protects against osteoarthritis. Exp Ther Med 18: 1677-1684, 2019.

14. Livak KJ and Schmittgen TD: Analysis of relative gene expression data using real-time quantitative PCR and the 2(-Delta Delta C(T)) method. Methods 25: 402-408, 2001

15. Giudice A, Arra C and Turco MC: Review of molecular mechanisms involved in the activation of the Nrf2-ARE signaling pathway by chemopreventive agents. Vol. 647. Higgins P (ed). In: Transcription Factors. Methods in Molecular Biology (Methods and Protocols). Springer, Humana Press, Totowa, NJ. pp37-74, 2010.

16. Dong Y, Stewart T, Bai L, Li X, Xu T, Iliff J, Shi M, Zheng D, Yuan L, Wei T, et al: Coniferaldehyde attenuates Alzheimer's pathology via activation of Nrf2 and its targets. Theranostics 10: 179-200, 2020

17. Kim J, Xu M, Xo R, Mates A, Wilson GL, Pearsall AW IV and Grishko V: Mitochondrial DNA damage is involved in apoptosis caused by pro-inflammatory cytokines in human OA chondrocytes. Osteoarthritis Cartilage 18: 424-432, 2010.

18. Henrotin YE, Bruckner P and Pujol JP: The role of reactive oxygen species in homeostasis and degradation of cartilage. Osteoarthritis Cartilage 11: 747-755, 2003.
19. Henrotin Y, Kurz B and Aigner T: Oxygen and reactive oxygen species in cartilage degradation: Friends or foes? Osteoarthritis Cartilage 13: 643-654, 2005.

20. Cai D, Huff TW, Liu J, Yuan T, Wei Z and Qin J: Alleviation of cartilage destruction by sinapic acid in experimental osteoarthritis. Biomed Res Int 2019: 5689613, 2019.

21. Fernández P, Guillén MI, Gomar F and Alcaraz MJ: Expression of heme oxygenase-1 and regulation by cytokines in human osteoarthritic chondrocytes. Biochem Pharmacol 66: 2049-2052, 2003.

22. Rousset F, Nguyen MVC, Grange L, Morel F and Lardy B: Heme oxygenase-1 regulates matrix metalloproteinase MMP-1 secretion and chondrocyte cell death via Nox4 NADPH oxidase activity in chondrocytes. PLoS One 8: e66478, 2013.

23. Lee TS and Chau LY: Heme oxygenase-1 mediates the anti-inflammatory effect of interleukin-10 in mice. Nat Med 8: 240-246, 2002.

24. Lee IT, Luo SF, Lee CW, Wang SW, Lin CC, Chang CC, Chen YL, Chau LY and Yang CM: Overexpression of HO-1 protects against TNF-alpha-mediated airway inflammation by down-regulation of TNFR1-dependent oxidative stress. Am J Pathol 175: 519-532, 2009.

(i) (5) This work is licensed under a Creative Commons cc) Attribution-NonCommercial-NoDerivatives 4.0 International (CC BY-NC-ND 4.0) License. 